\title{
Propagating Fronts and the Center Manifold Theorem
}

\author{
J.-P. Eckmann ${ }^{1}$ and C. E. Wayne ${ }^{2}$ \\ ${ }^{1}$ Département de Physique Théorique, Université de Genève, CH-1211 Geneva 4, Switzerland \\ 2 Department of Mathematics, Pennsylvania State University, University Park, PA 16802, USA
}

Received February 12, 1990; in revised form July 19, 1990

\begin{abstract}
We prove the existence of propagating front solutions for the SwiftHohenberg equation (SH). Using the center manifold theorem we reduce the problem to a two dimensional system of ordinary differential equations. They describe stationary solutions and front solutions of the partial differential equation (SH). We identify the well-known "amplitude equation" as the lowest order approximation to the equation of motion on the center manifold.
\end{abstract}

\section{Introduction}

In this paper, we reconsider the existence problem for fronts in the SwiftHohenberg equation, which was studied in [CE1, CE2]. This equation is of the form

$$
\partial_{t} u(x, t)=\left(\alpha-\left(1+\partial_{x}^{2}\right)^{2}\right) u(x, t)-u^{3}(x, t) .
$$

It is known that for small positive $\alpha$ this equation has stationary solutions (i.e., time-independent solutions) which are periodic with period $\omega$, for $|\omega|$ close to 1 . If we define $\varepsilon>0$ by

$$
\left(\omega^{2}-1\right)^{2}+\varepsilon^{2}=\alpha
$$

then these solutions are of the form

$$
u(x)=S(x) \approx \frac{2}{\sqrt{3}} \varepsilon \cos (\omega x) .
$$

Furthermore, in [CE1], front solutions for Eq. (1.1) were defined as solutions of the form

$$
u(x, t)=W(x, x-c t)
$$

with the boundary conditions at infinity

$$
\lim _{y \rightarrow-\infty} W(x, y)=S(x), \lim _{y \rightarrow+\infty} W(x, y)=0 .
$$


These solutions look like a fixed envelope advancing in the laboratory frame and leaving a periodic pattern (looking like the stationary solution) behind.

The aim of the present paper is to give a conceptually simpler proof of the existence of these stationary solutions and front solutions, using the techniques of reduction to a (2-dimensional) center manifold. Early references dealing with the relation between center manifolds and amplitude equations are [CM1, CM2]. The use of the center manifold theorem is complicated by the fact that one is perturbing around a state in which all directions are "center" directions. (For another application in such a situation see [LW].) Thus, we need to use the fact that after perturbation, some directions are "more central" than others. (In this respect they are similar to the " $r$-hyperbolic" manifolds of [HPS].) This requires us to use some care in treating the conditions which govern the applicability of the center manifold theorem. Since none of the published versions of this theorem seemed to be applicable to our problem, a detailed rederivation of the center manifold theorem is included in Appendix A.

In order to describe informally the nature of the center manifold and the relevant dynamics in it, we use a Fourier decomposition. We write

$$
S(x)=\sum_{n \in \mathbf{Z}} S_{n} e^{-i n \omega x},
$$

and a corresponding decomposition of $W(x, y)$,

$$
W(x, y)=\sum_{n \in \mathbf{Z}} W_{n}(y) e^{-i n \omega x}
$$

Then the boundary conditions for the $W_{n}$ are

$$
\lim _{y \rightarrow-\infty} W_{n}(y)=S_{n}, \quad \lim _{y \rightarrow \infty} W_{n}(y)=0 .
$$

To simplify the discussion, assume $\omega=1$. We are interested in small, positive $\alpha$, and we assume

$$
\alpha=\varepsilon^{2} \alpha_{0}, \quad c=\varepsilon c_{0} .
$$

Here, $\alpha_{0}$ and $c_{0}$ are fixed constants, and $\varepsilon>0$ is a small parameter. It is well-known that in this parameter range, a multiscale analysis is adequate. The principal contribution to $W$ comes from $n= \pm 1$, and we reparametrize $W_{1}$ as

$$
U(\xi)=\varepsilon W_{1}(\varepsilon \xi) \text {. }
$$

Then $U$ approximately satisfies the "amplitude equation,"

$$
4 U^{\prime \prime}+c_{0} U^{\prime}+\alpha_{0} U-3 U|U|^{2}=0 .
$$

The two components, $U$ and $U^{\prime}$, of this ordinary differental equation form essentially the two coordinates of the center manifold. They define a two-dimensional (complex) dynamical system in the center manifold, whose fixed points correspond to the stationary solutions of (1.1) and whose saddle connections between these fixed points and the zero solution correspond to the front solutions. It should be noted that the dynamical system obtained in the center manifold depends on the speed $c$ which is imposed on the front. It should also be noted that the original Eq. (1.1) is translation invariant. This will imply a symmetry of the induced flow on the center manifold, namely covariance under multiplication by a phase. 
In Sect. 2, we reduce the problem to the center manifold and perform the necessary perturbative calculations, while the proof of a version of the center manifold theorem is relegated to Appendix A. In Sect. 3, we study the flow on the center manifold itself, transforming it to a more useful form. In Sect. 4, we show the existence of a saddle connection. It is interesting to note already at this point that the symmetries of the problem do not guarantee the existence of stationary solutions. All that follows is that Eq. (1.1) either has stationary periodic solutions or quasistationary convective solutions, that is, solutions of the form

$$
u(x, t)=f(x-c t)
$$

with $f$ a periodic function. On the other hand, this dichotomy shows beautifully how the convective instabilities can occur as a consequence of translation invariance. For completeness, we give in Appendix B a direct argument for the existence of stationary rather than convective solutions, cf. [CE1].

The main result of our analysis is

Theorem 1.1. For $c_{0}^{2}>16 \alpha_{0}>0$, and for $\varepsilon$ sufficiently small, there is an interval of frequencies $\omega$, of size $\mathcal{O}\left(\varepsilon^{v}\right), v>1$, centered at $\omega=1$, for which (1.1) has front solutions of frequency $\omega$, of the form (1.2). The amplitudes of these solutions are close to solutions of (1.3).

Remark. Our results could also be derived for other forms of the non-linearity in (1.1) (e.g. $u^{5}$ ). However, the dependence of various quantities on $\varepsilon$ would change.

\section{Reduction to a Center Manifold}

For fixed $\omega$, and in a frame moving with speed $c$ the differential equation for $W_{n}$ takes the form

$$
\left(\alpha+c \partial_{\xi}-\left(1+\left(-i \omega n+\partial_{\xi}\right)^{2}\right)^{2}\right) W_{n}(\xi)=\sum_{p+q+r=n} W_{p}(\xi) W_{q}(\xi) W_{r}(\xi)
$$

We can write this fourth order equation as a system of four first order equations, and we then view $\xi$ as the "dynamical variable." We shall label the variables as follows:

$$
X_{n j} \leftrightarrow \partial_{\xi}^{j} W_{n}, \quad j=0, \ldots, 3, \quad n \in \mathbf{Z} .
$$

The system of Eq. (2.1) takes then the form

$$
\partial_{\xi} X_{n}=M_{n} X_{n}+F_{n}(X)
$$

where the matrix $M_{n}$ is of the form

$$
M_{n}=\left(\begin{array}{cccc}
0 & 1 & 0 & 0 \\
0 & 0 & 1 & 0 \\
0 & 0 & 0 & 1 \\
A & B & C & D
\end{array}\right),
$$


with

$$
\begin{aligned}
& A=-\left(1-\mu^{2}\right)^{2}+\alpha, \\
& B=4 i \mu\left(1-\mu^{2}\right)+c, \\
& C=6 \mu^{2}-2, \\
& D=4 i \mu,
\end{aligned}
$$

and $\mu=\omega n$. The non-linear part is given by the vector

$$
F_{n}(X)=\left(\begin{array}{c}
0 \\
0 \\
0 \\
E_{n}
\end{array}\right),
$$

where

$$
E_{n}=-\sum_{p+q+r=n} W_{p} W_{q} W_{r}=-\sum_{p+q+r=n} X_{p_{0}} X_{q_{0}} X_{r_{0}}
$$

2.1. The Linear Operator. The calculations involving $M_{n}$ can be done easily by observing that the characteristic polynomial of $M_{n}$ is

$$
p_{n}(\lambda)=\lambda^{4}-D \lambda^{3}-C \lambda^{2}-B \lambda-A .
$$

We begin by studying the spectrum of $M_{n}$ for small values of $\alpha$ and $c$ by performing perturbation theory in $\alpha$ and $c$. When $\alpha$ and $c$ are zero, the characteristic polynomial factors as $p_{n}(\lambda)=(\lambda-i(\mu+1))^{2}(\lambda-i(\mu-1))^{2}$ and the spectrum of $M_{n}$ consists of the double eigenvalues $i(\mu \pm 1)$ with eigenvectors

$$
\left(\begin{array}{c}
1 \\
i(\mu \pm 1) \\
-(\mu \pm 1)^{2} \\
-i(\mu \pm 1)^{3}
\end{array}\right)=v_{\mu}^{ \pm}
$$

and a nilpotent part

$$
\left(\begin{array}{c}
0 \\
1 \\
2 i(\mu \pm 1) \\
-3(\mu \pm 1)^{2}
\end{array}\right)=w_{\mu}^{ \pm}
$$

The action of $M_{n}$ is described by

$$
\begin{aligned}
M_{n} v_{\mu}^{ \pm} & =i(\mu \pm 1) v_{\mu}^{ \pm}, \\
M_{n} w_{\mu}^{ \pm} & =i(\mu \pm 1) w_{\mu}^{ \pm}+v_{\mu}^{ \pm} .
\end{aligned}
$$

Thus, we see that for $\alpha=0, c=0$, the linear part of the problem has purely imaginary spectrum.

We shall now study how this spectrum evolves as the parameters are varied. We shall see that for $n= \pm 1$ the spectrum will leave the imaginary axis by an amount which is an order of magnitude smaller than for the other values of $n$.

We consider the case of small $\alpha$ and $c$. In order to make perturbative statements 
we set

$$
\alpha=\varepsilon^{2} \alpha_{0}, \quad c=\varepsilon c_{0} .
$$

For simplicity, we set $\omega=1$; see below for a generalization to $\omega \neq 1$. It is useful to define

$$
\Delta=\sqrt{c_{0}^{2}-16 \alpha_{0}} .
$$

The characteristic polynomial may now be written as

$$
p_{n}(\lambda)=(\lambda-i(\mu+1))^{2}(\lambda-i(\mu-1))^{2}-c_{0} \varepsilon \lambda-\alpha_{0} \varepsilon^{2},
$$

where $\mu=n \omega=n$. If $\varepsilon \mu$ is small, we may write $\lambda=i(\mu \pm 1)+\delta$, and solve approximately for $\delta$, and one finds the asymptotic formulae

$$
\begin{aligned}
& \lambda_{\mu, \pm}^{+}= \begin{cases}\varepsilon\left(-c_{0} \pm \Delta\right) / 8+\mathcal{O}\left(\varepsilon^{2}\right) & \text { when } n=-1 \\
i(\mu+1) \pm \varepsilon^{1 / 2} i^{3 / 2} \sqrt{c_{0}(\mu+1)} / 2+\mathcal{O}(\varepsilon) & \text { when } n \neq-1\end{cases} \\
& \lambda_{\mu, \pm}^{-}= \begin{cases}\varepsilon\left(-c_{0} \pm \Delta\right) / 8+\mathcal{O}\left(\varepsilon^{2}\right) & \text { when } n=1 \\
i(\mu-1) \pm \varepsilon^{1 / 2} i^{3 / 2} \sqrt{c_{0}(\mu-1)} / 2+\mathcal{O}(\varepsilon) & \text { when } n \neq 1,\end{cases}
\end{aligned}
$$

for the eigenvalues. If, on the other hand, $\varepsilon \mu>\tilde{c}$ for some small constant $\tilde{c}$, then one shows easily that there exists some small positive constant $r_{1}$ such that $\left|\operatorname{Re} \lambda_{\mu, \pm}^{ \pm}\right|>r_{1}$. We assume now $\varepsilon>0, \alpha_{0}>0$ and $c_{0}^{2}>16 \alpha_{0}$. Then $\Delta$ is real. (The other case for $c_{0}$ corresponds to fronts which are slower than the minimal speed for which the amplitude equation has positive solutions.) We see that for $\omega=1$,

$$
\begin{aligned}
& \operatorname{Re} \lambda_{-1, \pm}^{+}=\varepsilon\left(-c_{0} \pm \Delta\right) / 8+\mathcal{O}\left(\varepsilon^{2}\right), \\
& \operatorname{Re} \lambda_{+1, \pm}^{-}=\varepsilon\left(-c_{0} \pm \Delta\right) / 8+\mathcal{O}\left(\varepsilon^{2}\right) .
\end{aligned}
$$

For all other choices of $n \in \mathbf{Z}$ and of the sign $s \in\{+,-\}$, we have

$$
\operatorname{Re} \lambda_{n, s}^{ \pm}=\mathcal{O}\left(\varepsilon^{1 / 2}\right) \text {. }
$$

This means that $\lambda_{-1, \pm}^{+}$and $\lambda_{+1, \pm}^{-}$are "more central" than all other eigenvalues. Note that if $\omega^{2}-1=\omega_{0} \varepsilon$, the preceding observations remain valid.

2.2. The Function Space. Let $\mathscr{E}_{0}$ denote the direct sum $\bigoplus_{n=0}^{\infty} \mathbf{C}^{4}$. If $X \in \mathscr{E}_{0}$, we denote by $X_{n j}, n \geqq 0, j=0, \ldots, 3$, the components of $X$ in the natural decomposition of $\bigoplus_{n=0}^{\infty} \mathbf{C}^{4}$. We denote $\mathscr{E}$ the subspace of $\mathscr{E}_{0}$ in which $X_{0 j}, j=0, \ldots, 3$ are real.

There will be a one-to-one map $I_{y}, y \in \mathbf{R}$ from the space of real, sufficiently differentiable functions of form

$$
W(x, y)=\sum_{n \in \mathbf{Z}} e^{-i n \omega x} W_{n}(y),
$$

into $\mathscr{E}$. It is defined by

$$
I_{y}(W)_{n j}=\left.\partial_{z}^{j} W_{n}(z)\right|_{z=y} .
$$

Note that $W_{-n}(y)=\bar{W}_{n}(y)$, so that the $I_{y}(W)_{n j} \in \mathbf{C}$ for $n>0$, and the $I_{y}(W)_{0 j} \in \mathbf{R}$ uniquely determine $W$. 
We make $\mathscr{E}_{0}$ into a Hilbert space by defining the inner product

$$
\langle X, Y\rangle_{l}=\sum_{n}\left(1+n^{2}\right)^{l} \bar{X}_{n j} Y_{n j}
$$

and setting $H_{\mathbf{C}}^{l}\left(\mathscr{E}_{0}\right)=\left\{X \in \mathscr{E}_{0} \mid\langle X, X\rangle_{l}<\infty\right\}$.

2.3. The Nonlinearity. We consider the nonlinearity $F: \mathscr{E}_{0} \rightarrow \mathscr{E}_{0}$, whose components are given in (2.3). The following estimate shows that $F$ is well-behaved.

Lemma 2.1. If $l>1 / 2$, then $F$ is a continuously differentiable (and hence analytic) function from $H_{\mathbf{C}}^{l}\left(\mathscr{E}_{0}\right) \rightarrow H_{\mathbf{C}}^{l}\left(\mathscr{E}_{0}\right)$.

This is a standard result in Sobolev space theory. It follows from the fact that $H_{C}^{l}$ is a Banach algebra and an easy calculation, which we do not reproduce. Thus we have the

Corollary 2.2. If $l>1 / 2$, then there exists a constant $c_{F, l}>0$, such that

$$
\|F(x)\|_{l} \leqq c_{F, l}\|x\|_{l}^{3} .
$$

2.4. The Flow on the Center Manifold. We begin the reduction of our system of equations to the center-manifold by studying in more detail the eigenvectors and eigenvalues of the linearized piece which correspond to the "center" directions of the equations. By the center directions, we mean the subspace of $\mathscr{E}$ corresponding to the eigenvalues whose real part are of order $\mathcal{O}(\varepsilon)$.

We first note that the submatrices $M_{n}$ of the linearized operator leave a fourdimensional subspace of $\mathscr{E}$ invariant. We refer to this subspace as the " $n$th sector." We note further than the center directions are confined to the first sector.

Given the matrix $M_{1}$, we will use the fact, noted in Appendix $\mathrm{C}$, that if $\phi$ is the eigenvector corresponding to eigenvalue $\lambda$, and if $M_{1}^{\dagger}$, is the adjoint of $M_{1}$, with $\psi$ the adjoint eigenvector corresponding to the eigenvalue $\bar{\lambda}$, then $\langle\psi, \phi\rangle=p_{1}^{\prime}(\lambda)$, the derivative of the characteristic function of $M_{1}$. The adjoint is calculated with respect to the usual inner product, namely, $\langle\psi, \phi\rangle=\sum_{i} \bar{\psi}_{i} \phi_{i}$, and we normalize the eigenvectors so that the first component of $\phi$ and the last component of $\psi$ are 1 .

Because of the definition of the subspace $\mathscr{E}$ on which we work, there are only two center directions, corresponding to the eigenvalues $\lambda_{+1, \pm}^{-}$. We will henceforth refer to these eigenvalues as $\lambda_{ \pm}$when there is no possibility of confusion, and to their corresponding eigenvectors and adjoint eigenvectors as $\phi_{ \pm}$and $\psi_{ \pm}$.

In deriving the reduced equations on the center manifold we follow the method of Kirchgässner[K] and Mielke[M]. We introduce the projection operators

$$
\mathbf{P}=c_{+}\left|\phi_{+}\right\rangle\left\langle\psi_{+}\left|+c_{-}\right| \phi_{-}\right\rangle\left\langle\psi_{-}\right| ; \mathbf{P}^{\perp}=\mathbf{1}-\mathbf{P} .
$$

In this definition $c_{ \pm}$are normalization constants chosen so that $c_{ \pm}\left\langle\psi_{ \pm}, \phi_{ \pm}\right\rangle=1$. Note further that $\left\langle\psi_{\mp}, \phi_{ \pm}\right\rangle=0$, since $\lambda_{+} \neq \lambda_{-}$when $\alpha_{0} \neq 0$ and $\varepsilon$ is sufficiently small.

We now rewrite the variable $X$ in our differential equation as $X=w+w^{\perp}$, where $w=\mathbf{P} X$, and $w^{\perp}=\mathbf{P}^{\perp} X$. Note that $w$ is always contained in the first sector. The new equations are:

$$
\partial_{\xi} w=M w+\mathbf{P} F\left(w+w^{\perp}\right)
$$




$$
\partial_{\xi} w^{\perp}=M w^{\perp}+\mathbf{P}^{\perp} F\left(w+w^{\perp}\right) .
$$

The center manifold theorem (see Appendix A) implies that there exists an invariant manifold of the form $(w, h(w))$, with $h$ a $\mathscr{C}^{1}$ map from $\mathbf{P} \mathscr{E}_{0}$ to $\mathbf{P}^{\perp} \mathscr{E}_{0}$ defined a neighborhood of the origin of size $\mathcal{O}\left(\varepsilon^{3 / 4+\gamma}\right)$, for any $\gamma>0$, provided $\varepsilon$ is sufficiently small. Since we are interested in solutions of size $\mathcal{O}(\varepsilon)$ this is a sufficiently large neighborhood for our purpose. If we substitute $h(w)$ for $w^{\perp}$ in (2.8), we obtain, from the invariance under the flow, the equation

$$
h^{\prime}(w) \cdot \partial_{\xi} w=M h(w)+\mathbf{P}^{\perp} F(w+h(w)) .
$$

We now substitute for $\partial_{\xi} w$ from (2.7) and obtain

$$
h^{\prime}(w) M w-M h(w)=-h^{\prime}(w) \mathbf{P} F(w+h(w))+\mathbf{P}^{\perp} F(w+h(w)) .
$$

If we now assume that $h(w) \approx \mathcal{O}\left(w^{m}\right)$ near the origin, then the left-hand side of this equation is $\mathcal{O}\left(w^{m}\right)$, while the right-hand is $\mathcal{O}\left(w^{m+2}\right)+\mathcal{O}\left(w^{3}\right)$. (This uses the fact that $F(w) \approx \mathcal{O}\left(w^{3}\right)$.) Hence, we may choose $h(w) \approx \mathcal{O}\left(w^{3}\right)$ near the origin. Thus, if we ignore terms of order $\mathcal{O}\left(w^{4}\right)$ and higher we obtain approximate equations of motion in the center manifold, just by setting $w^{\perp}=0$ in (2.7).

We now introduce coordinates $x_{ \pm}$on the center manifold. We write $w=x_{+} \phi_{+}+x_{-} \phi_{-}$and recall that $X=w+w^{\perp}$, so that when $w^{\perp}=0, x_{+}+x_{-}=$ $X_{10}$. In order to calculate the form of the nonlinear term in the $x$ variables, we note that

$$
\begin{aligned}
\mathbf{P} F(w) & =c_{+}\left|\phi_{+}\right\rangle\left\langle\psi_{+}, F(w)\right\rangle+c_{-}\left|\phi_{-}\right\rangle\left\langle\psi_{-}, F(w)\right\rangle \\
& =c_{+}\left|\phi_{+}\right\rangle E_{1}(w)+c_{-}\left|\phi_{-}\right\rangle E_{1}(w) .
\end{aligned}
$$

The last of these equalities came from the explicit formulae for $\psi_{ \pm}$and $F(w)$.

Rewriting (2.7) in terms of the $x$ 's, using this information we obtain

$$
\begin{aligned}
& \dot{x}_{+}=\lambda_{+} x_{+}-3 c_{+}\left(x_{+}+x_{-}\right)\left|x_{+}+x_{-}\right|^{2}, \\
& \dot{x}_{-}=\lambda_{-} x_{-}-3 c_{-}\left(x_{+}+x_{-}\right)\left|x_{+}+x_{-}\right|^{2} .
\end{aligned}
$$

\section{Identification with the Amplitude Equation}

We now work out an expansion for these quantities in powers of $\varepsilon$. Note that we are assuming throughout $c_{0}^{2}>16 \alpha_{0}$, so that $\Delta$ is real. For our purposes it is only necessary to use expansions up to order $\mathcal{O}(\varepsilon)$. In Sect. 2.1, we already computed $\lambda_{ \pm}$:

$$
\lambda_{ \pm}=\varepsilon\left(-c_{0} \pm \Delta\right) / 8+\mathcal{O}\left(\varepsilon^{2}\right)
$$

Using the remark made earlier we have $c_{ \pm}=1 /\left\langle\psi_{ \pm}, \phi_{ \pm}\right\rangle=1 / p_{1}^{\prime}\left(\lambda_{ \pm}\right)$. Using the expression (2.4) for $p_{1}(\lambda)$, and the asymptotic formula for $\lambda_{ \pm}$from above, we find that

$$
c_{ \pm}=\frac{\mp 1}{\varepsilon \Delta}(1+\mathcal{O}(\varepsilon)) \text {. }
$$

We now change coordinates in (2.9). We choose

$$
\chi=\left(x_{+}+x_{-}\right), \quad \eta=\left(x_{+}-x_{-}\right) .
$$


Neglecting terms of higher order, this leads to

$$
\begin{aligned}
& \dot{\chi}=\dot{x}_{+}+\dot{x}_{-}=-\varepsilon \frac{c_{0}}{8} \chi+\varepsilon \frac{\Delta}{8} \eta, \\
& \dot{\eta}=\dot{x}_{+}-\dot{x}_{-}=\varepsilon \frac{\Delta}{8} \chi-\varepsilon \frac{c_{0}}{8} \eta+\frac{6}{\varepsilon \Delta} \chi\left|\chi^{2}\right| .
\end{aligned}
$$

We next rescale the "time:" Define

Then

$$
\chi(t)=\varepsilon u(\varepsilon t), \quad \eta(t)=\varepsilon v(\varepsilon t) .
$$

$$
\dot{\chi}(t)=\varepsilon^{2} \dot{u}(\varepsilon t), \quad \chi^{3}(t)=\varepsilon^{3} u^{3}(\varepsilon t),
$$

and the equations take the form

$$
\begin{aligned}
& \dot{u}=-\frac{c_{0}}{8} u+\frac{\Delta}{8} v, \\
& \dot{v}=\frac{\Delta}{8} u-\frac{c_{0}}{8} v+\frac{6}{\Delta} u\left|u^{2}\right| .
\end{aligned}
$$

Our next transformation is

$$
u=q, \quad v=\frac{c_{0} q}{\Delta}+\frac{8 p}{\Delta} .
$$

Then the equations take the form

$$
\begin{aligned}
& \dot{q}=p, \\
& \dot{p}=\frac{1}{4}\left(-\alpha_{0} q-c_{0} p+3 q|q|^{2}\right) .
\end{aligned}
$$

Clearly, the system (3.2) is equivalent to (1.3).

\section{Stationary Solutions and Fronts}

The vector field described by (3.2) is very well studied in the literature, see for example [AW]. We need here a discussion of a slightly perturbed system in the complex domain. Although Eq. (3.2) could be viewed as a real equation, the correction terms of higher order will force the solution to acquire imaginary components even if the initial data at $x=-\infty$ are real. This phenomenon describes in fact the corrections of the positions of the nodes of the solution when the amplitudes change. Equation (3.2) has a fixed point at $p=q=0$, and a circle of fixed points at $p=0,|q|=\left(\alpha_{0} / 3\right)^{1 / 2}$. (We assume always $\alpha_{0}>0$.) We need to know what happens to these fixed points when we perturb slightly Eq. (3.2). It will be seen that the fixed point at 0 is hyperbolic and that the circle of fixed points is normally hyperbolic. This will imply that under a small perturbation, the fixed point at zero persists, and the circle remains an invariant circle. However, no abstract argument guarantees that it remains a circle of fixed points. We will provide a separate argument in Appendix B, which shows that the invariant circle 
is made up of fixed points. We stress again that the other alternative is physically not uninteresting and has been observed in other, realistic examples, cf. [CE2]. It corresponds to convective instabilities.

4.1. Stationary or Convective Solutions. We now study (3.2) as an equation in $\mathbf{C}^{2}$. Writing out the equations for the real and imaginary parts of $p$ and $q$, we see that the linearization of the vector field at the origin has two double eigenvalues, $\frac{-c_{0} \pm \sqrt{c_{0}^{2}-16 \alpha_{0}}}{8}$, (which both have real part less than zero), and the linearization at $q=\sqrt{\alpha_{0} / 3}$ has eigenvalues $0,-c_{0} / 4$, and $\frac{-c_{0} \pm \sqrt{c_{0}^{2}+32 \alpha_{0}}}{8}$. Note that here we have one unstable direction, one neutral direction, corresponding to motion along the circle of fixed points, and two stable directions. Thus, the circle is normally hyperbolic cf. [HPS].

We now study what happens to the fixed point and the circle as we perturb the vector field. Let $\mathbf{X}_{0}$ denote the vector field in (3.2), and let $\mathbf{X}_{\varepsilon}$ be the $\mathscr{C}^{1}$ small perturbation of $\mathbf{X}_{0}$ obtained by changing the parameters to $\varepsilon>0$. Then we have:

\section{Lemma 4.1.}

$\mathrm{X} 1: \mathbf{X}_{\varepsilon}$ is covariant under the transformation $q \rightarrow e^{i \phi} q$ and $p \rightarrow e^{i \phi} p$, and

$\mathrm{X} 2: \mathbf{X}_{\varepsilon}$ has a fixed point which approaches $q=\sqrt{\alpha_{0} / 3}, p=0$ as $\varepsilon$ approaches zero.

Proof. The covariance is obvious from the translation invariance of (1.1). We show in Appendix B that $\mathbf{X}_{\varepsilon}$ satisfies X2.

Remark. Note that if $\mathbf{X}_{\varepsilon}$ has a non-zero fixed point, then by $\mathrm{X} 1$, it has a whole circle of fixed points.

4.2. Front Solutions. The circle of fixed points with $|q|>0$ corresponds to stationary solutions, and any two of these solutions differ only by a phase (which corresponds to translation in $x$ for the original Eq. (1.3)). The point $p=q=0$ corresponds to the zero solution of Eq. (1.3). For the unperturbed system, (3.2), a phase space analysis (see $[\mathrm{AW}]$ ) shows that for every $q$ on the circle $|q|^{2}=\alpha_{0} / 3$, there is a saddle connection, tangent to the unstable direction at that point, which connects it to the point $p=q=0$. The connections for different "initial points" $q$ are again relation by a phase, and if $q^{2}=\alpha_{0} / 3$, they are real. We now show that these front solutions persist when we perturb the Eq. (3.2).

Lemma 4.2. Under the conclusions of Lemma 4.1, $\mathbf{X}_{\varepsilon}$ has a hyperbolic fixed point at $p=0, q=0$, and a circle of fixed points near $|q|^{2}=\alpha_{0} / 3$. Furthermore, $\mathbf{X}_{\varepsilon}$ has a family of front solutions (related to one another via $q \rightarrow e^{i \phi} q$ and $p \rightarrow e^{i \phi} p$ ) which are saddle connections between the circle of fixed points and the origin.

Proof. We begin by considering a neighborhood of the circle $|q|^{2}=\alpha_{0} / 3$ in $\mathbf{C}^{2}$. Note that this circle is a normally hyperbolic, invariant set for (3.2). From the theory of such sets (see [HPS], for example), we know that $\mathbf{X}_{\varepsilon}$ will have a normally hyperbolic, invariant circle nearby. Furthermore this will be the only invariant set in a neighborhood of the circle, $|q|^{2}=\alpha_{0} / 3$, so the fixed point obtained in $\mathrm{X} 2$ above must lie on this circle. But then by the remark following X2, the whole circle must consist of fixed points. For $\varepsilon$ sufficiently small, the fixed points will 
have one unstable, one neutral, and two stable directions, and on a sufficiently small neighborhood of this (circle of) fixed points, the flow will be topologically conjugate to the flow of $\mathbf{X}_{0}$. Let the conjugating homeomorphism be $h_{1, \varepsilon}$.

Let $\mathscr{F}_{0}$ be the front solution of $\mathbf{X}_{0}$ which connects $q=\sqrt{\alpha_{0} / 3}$ with the origin. If one considers any compact segment of $\mathscr{F}_{0}$, excluding the points $q=\sqrt{\alpha_{0} / 3}$, and the origin, then $\mathbf{X}_{0}$ is non-singular in a neighborhood and hence is topologically conjugate to $\mathbf{X}_{\varepsilon}$ via a homeomorphism $h_{2, \varepsilon}$, provided $\varepsilon$ and the neighborhood are sufficiently small (see e.g. [A, Sect. 32]). We can choose the segment of $\mathscr{F}_{0}$ such that the domains of $h_{1, \varepsilon}$ and $h_{2, \varepsilon}$ intersect, and (by a choice of origin) we can insure that $h_{1, \varepsilon}\left(\mathscr{F}_{0}\right)=h_{2, \varepsilon}\left(\mathscr{F}_{0}\right)$ on the intersection of the domains. (Note that if $h_{1, \varepsilon}\left(\mathscr{F}_{0}\right)$ and $h_{2, \varepsilon}\left(\mathscr{F}_{0}\right)$ agree at a single point they agree at all points since $h_{i, \varepsilon}, i=1,2$ map trajectories of $\mathbf{X}_{0}$ to trajectories of $\mathbf{X}_{\varepsilon}$.) Finally, we note that since the origin is an attractive fixed point for $\mathbf{X}_{\varepsilon}$, we can choose the segment of $\mathscr{F}_{0}$ around which we construct $h_{2, \varepsilon}$ to be such that $h_{2, \varepsilon}\left(\mathscr{F}_{0}\right)$ intersects the basin of attraction of the origin. We then define a trajectory $\mathscr{F}_{\varepsilon}$ as follows:

i) $\mathscr{F}_{\varepsilon}$ is the image of $\mathscr{F}_{0}$ under $h_{i, \varepsilon} ; i=1,2$ on the domain of these homeomorphisms.

ii) Once $\mathscr{F}_{\varepsilon}$ intersects the basin of attraction of the origin, we extend it via the flow associated with $\mathbf{X}_{\varepsilon}$.

Note that $\mathscr{F}_{\varepsilon}$ is asymptotic to the circle of fixed points as $t \rightarrow-\infty$. As $t \rightarrow \infty$, it is asymptotic to the origin. Thus, $\mathscr{F}_{\varepsilon}$ is a front solution for $\mathbf{X}_{\varepsilon}$ and once we have one front, the covariance of the equations under $q \rightarrow e^{i \phi} q$ and $p \rightarrow e^{i \phi} p$ allows us to construct a whole family of such trajectories. Note, however, that the solution starting at $p=0, q>0, q$ real is not necessarily real any longer. The phases of $p$ and $q$ one observes now describe the displacement of nodes in (1.3) which is caused by the constriction of the amplitude, while the covariance of the whole vector field describes the overall translation invariance of the problem. We have thus shown the following

Theorem 4.3. Given $c_{0}^{2}>16 \alpha_{0}>0$, there is an $\varepsilon_{0}>0$ such that for all $\varepsilon$ in $\left(0, \varepsilon_{0}\right)$ Eq. (1.1) has stationary solutions and front solutions of frequency $\omega=1$, and of the form (1.2). The amplitudes of these solutions are close to saddle connections for Eq. (3.2).

Note that all our considerations above still apply if we replace $\omega=1$, with any $\omega$ for which $\left|\omega^{2}-1\right|<\varepsilon^{\nu}$, for some $v>1$. Thus we have the

Corollary 4.4. Given $c_{0}^{2}>16 \alpha_{0}>0$, there is an $\varepsilon_{0}>0$ such that for all $\varepsilon$ in $\left(0, \varepsilon_{0}\right)$ Eq. (1.1) has stationary solutions and front solutions of frequency $\omega$, for all $\left|\omega^{2}-1\right|<\varepsilon^{v}$, where $v>1$. The amplitudes of these solutions are close to saddle connections for Eq. (3.2).

This completes the proof of Theorem 1.1.

\section{Appendix A. The Center Manifold Theorem}

The theorem we present below is well-known. (See for e.g. [MM], where a proof similar to the one below is presented.) However, we will nevertheless give a complete 
proof because we need to know in detail how the various constants in the theorem depend on $\varepsilon$, and that does not seem to be available in the literature.

We consider a differential equation on the Banach space $\mathscr{E}$, and we assume that $\mathscr{E}=\mathscr{E}^{c} \oplus \mathscr{E} \oplus \oplus \mathscr{E}^{\mathfrak{c}}$, which we refer to respectively as the center, the stable, and the unstable subspaces. Let $\left(z^{c}, z^{s}, z^{u}\right)$ be coordinates for these subspaces. We write the differential equation in the form:

$$
\begin{aligned}
& \frac{d z^{c}}{d t}=A^{c} z^{c}+f^{c}\left(z^{c}, z^{s}, z^{u}\right), \\
& \frac{d z^{s}}{d t}=A^{s} z^{s}+f^{s}\left(z^{c}, z^{s}, z^{u}\right), \\
& \frac{d z^{u}}{d t}=A^{u} z^{u}+f^{u}\left(z^{c}, z^{s}, z^{u}\right) .
\end{aligned}
$$

Any combination of the indices $c, s, u$, will refer to the direct sum of the corresponding subspaces, and the corresponding norm will be the maximum. For example, $\mathscr{E}^{c s}=\mathscr{E}^{c} \oplus \mathscr{E}^{s}$ with the norm $\left\|z^{c} \oplus z^{s}\right\|_{c s}=\max \left(\left\|z^{c}\right\|_{c},\left\|z^{s}\right\|_{s}\right)$. Define $B_{r}^{c}$ to be the ball of radius $r$, centered at the origin in $\mathscr{E}^{c}$, with $B_{r}^{u}$ and $B_{r}^{s}$ analogously defined. We also need $B_{r}^{c s}=B_{r}^{c} \oplus B_{r}^{s}$ and similar combinations. Denote by $P_{c}, P_{s}$ and $P_{u}$ the projections onto the components of $\mathscr{E}$.

We make the following hypotheses.

H1: The linear operators $A^{s}$ and $A^{u}$ define continuous semigroups on $\mathscr{E}^{s}$ and $\mathscr{E}^{u}$, for $t \geqq 0$, and $t \leqq 0$, respectively. Furthermore, we assume that there exist positive constants $\bar{\lambda}_{s}, \lambda_{u}$, and $D$, such that $\sup \max \left(e^{\lambda_{s} t}\left\|e^{A^{s} t}\right\|_{s}, e^{\lambda_{u} t}\left\|e^{-A^{u_{t}}}\right\|_{u}\right) \leqq D$. $t \geqq 0$

H2: The linear operator $A^{c}$ defines a flow on $\mathscr{E}^{c}$, with $\left\|e^{A^{c} t} z^{c}\right\|_{c} \leqq a\left(1+|t|^{k}\right)\left\|z^{c}\right\|_{c}$ for all $t \in \mathbf{R}$.

H3: The functions $f^{c}$, and $f^{s}$, and $f^{u}$ are Lipschitz functions from $\mathscr{E}$ to $\mathscr{E}^{c}, \mathscr{E}^{s}$, and $\mathscr{E}^{u}$, respectively. The Lipschitz constants of these three functions on the ball of radius $r$ about the origin in $\mathscr{E}$ will be denoted by $l_{c}(r), l_{s}(r)$, and $l_{u}(r)$. By this we mean, e.g.,

$$
\left\|f^{s}(z)-f^{s}(\tilde{z})\right\|_{s} \leqq l_{s}(r)\|z-\tilde{z}\|_{c s u},
$$

for all $z, \tilde{z} \in B_{r}^{c s u}$. We require that all three functions $\left(l_{s}\right.$ etc.) vanish as $r \rightarrow 0$.

Theorem A.1. Assume that there exists a constant $\sigma>1$, and positive constants $\beta$ and $r$ such that the inequalities $\mathrm{C1}^{\prime}-\mathrm{C}^{\prime}$ below hold. Then there exists a Lipschitz function $h$ defined on some neighborhood, $U^{c}$, of the origin in $\mathscr{E}^{c}$ and mapping $U^{c}$ to $\mathscr{E}^{s} \oplus \mathscr{E}^{u}$. Furthermore, $h(0)=0$ and the graph of $h$ is left invariant by $(A .1)$. If the non-linearity $\left(f^{c}, f^{s}, f^{u}\right)$ in $(A .1)$ is $\mathscr{C}^{m+1}, 1 \leqq m<\infty$, if $\mathscr{E}$ has the $\mathscr{C}^{m+1}$ extension property, and if ' $\mathrm{C}^{\prime}$ ' below holds, then the function $h$ is $\mathscr{C}^{m}$, and the mth derivative is Lipschitz, possibly on a smaller neighborhood $\tilde{U}^{c}$.

Remark. A Banach space $\mathscr{E}$ has the $\mathscr{C}^{m}$ extension property if there exists a function $\chi \in \mathscr{C}^{m}(\mathscr{E}, \mathbf{R})$ such that $\chi(z)=1$ if $\|z\|<1 / 2$, and $\chi(z)=0$ if $\|z\|>1$. If $\mathscr{E}$ is finite dimensional, or a Hilbert space then it has the $\mathscr{C}^{m}$ extension property for all $m=1,2, \ldots, \infty$. 
Remark. One could assume that the vector field $f^{c, s, u}$ in Theorem A.1 was only $C^{m}$, with Lipschitz $m$ th derivative.

Proof of Theorem A.1. We begin by describing the construction of the center-stable manifold. The construction is very similar to that of [CE1], modified only to take account of the presence of the "center" directions (cf. [M]). We look for an invariant manifold, $W^{c s}$, which is the graph of a function $h: \mathscr{E}^{c s} \rightarrow \mathscr{E}^{n}$. To simplify notation, we denote points in $\mathscr{E}^{c s}$ by $\xi$, and let their projections onto $\mathscr{E}$, and $\mathscr{E}^{s}$ be denoted by $\xi^{c}$ and $\xi^{s}$ respectively. Let $\Psi_{t}^{h}$ be the projection onto $\mathscr{E}^{c s}$ of the flow on the graph of $h$.

Then formally, $\Psi_{t}^{h}$ satisfies

$$
\Psi_{t}^{h}=e^{A^{c s t}}+\int_{0}^{t} d \tau e^{A^{c s}(t-\tau)} f_{h}^{c s} \circ \Psi_{\tau}^{h} .
$$

Here, $A^{c s}=A^{c} \oplus A^{s}$, and

$$
f_{h}^{c s}(\xi)=\left(\begin{array}{l}
f^{c}(\xi, h(\xi)) \\
f^{s}(\xi, h(\xi))
\end{array}\right)
$$

In order for $W^{c s}$ to remain invariant under the flow $\phi_{t}$ we must have

$$
\phi_{t}(\xi, h(\xi))=\left(\begin{array}{c}
\Psi_{t}^{h}(\xi) \\
h\left(\Psi_{t}^{h}(\xi)\right)
\end{array}\right)
$$

from which it follows that

$$
h=-\int_{0}^{\infty} d \tau e^{-A_{\tau}^{u_{\tau}}} f_{h}^{u_{\circ}} \Psi_{\tau}^{h}
$$

We note that the calculations leading to (A.2) and (A.3) are formal ones - for instance, the flow $\phi_{t}$ will in general not be defined for all initial conditions in a neighborhood of the origin. We will prove that the calculations make sense by first showing that given $h$, (A.2) has a solution which we then substitute into the right-hand side of (A.3). We then consider the right-hand side of (A.3) as defining a transformation of the function $h$, which we then prove has a fixed point. That fixed point defines our center-stable manifold.

We begin by defining the spaces in which $h$ and $\Psi^{h}$ will be shown to lie. The space for $h$ is given by

$$
H_{\sigma}=\left\{h: \mathscr{E}^{c s} \rightarrow \mathscr{E}^{u} \mid h(0)=0,\|h(\xi)-h(\tilde{\xi})\|_{u} \leqq \sigma\|\xi-\tilde{\xi}\|_{c s}, \forall \xi, \tilde{\xi} \in \mathscr{E}^{c s}\right\} .
$$

If $h$ and $\tilde{h}$ are elements of $H_{\sigma}$, we define the Lipschitz metric

$$
d_{H}(h, \tilde{h})=\sup _{\substack{\xi \in \mathscr{E}^{c s} \\ \xi \neq 0}} \frac{\|h(\xi)-\tilde{h}(\xi)\|_{u}}{\|\xi\|_{c s}} .
$$

We next define the space in which the map $\Psi^{h}$ is going to live. Due to the bad control over the center direction, we will allow for a small exponential divergence, (cf. Mielke[M].) Fix $\beta>0$ (small). We require that $\beta<\min \left(\lambda_{s}, \lambda_{u}\right)$. 
Define

$$
\begin{gathered}
K_{\sigma, \beta}=\left\{\psi: \mathbf{R}^{+} \times \mathscr{E}^{c s} \rightarrow \mathscr{E}^{c s} \mid \psi_{0}(\xi)=\xi, \text { for all } \xi \in \mathscr{E}^{c s}\right. \\
\psi_{t}(0)=0, \text { for all } t \geqq 0, \\
\left.\left\|\psi_{t}(\xi)-\psi_{t}(\tilde{\xi})\right\|_{c s} \leqq \sigma e^{\beta t}\|\xi-\tilde{\xi}\|_{c s}, \text { for all } \xi, \tilde{\xi} \in \mathscr{E} c s\right\}
\end{gathered}
$$

We also define the Lipschitz norm

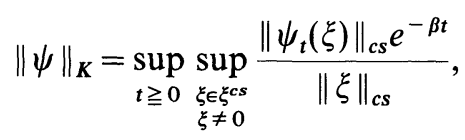

and the induced Lipschitz metric on $K_{\sigma, \beta}$,

$$
d_{K}(\psi, \tilde{\psi})=\|\psi-\tilde{\psi}\|_{K}
$$

Remark. $H_{\sigma}$ and $K_{\sigma, \beta}$ are complete metric spaces with respect to $d_{H}$ and $d_{K}$.

The construction of center manifolds makes the use of a cutoff necessary. We do not assume that $f$ itself has a restricted domain, but work instead with an explicit cutoff function $\chi$. This has the advantage of making the bounds on Lipschitz constants more explicit. We choose a smooth function $\chi_{0}: \mathbf{R} \rightarrow \mathbf{R}$, satisfying $0 \leqq \chi_{0} \leqq 1, \chi_{0}(x)=1$ when $|x| \leqq 1 / 2, \chi_{0}(x)=0$ when $|x|>1$. We assume for definiteness that the derivative satisfies $\left|\chi_{0}^{\prime}(z)\right| \leqq 3$. Let $z=\left(z^{c}, z^{s}, z^{u}\right)$. We then define $\chi(z)=\chi_{0}(\|z\|)$.

For $r$ a positive real number, we define the functions

$$
\begin{aligned}
& g^{s}(z)=f^{s}(z) \cdot \chi(z / r), \\
& g^{c}(z)=f^{c}(z) \cdot \chi(z / r), \\
& g^{u}(z)=f^{u}(z) \cdot \chi(z / r) .
\end{aligned}
$$

Then $g^{s}, g^{c}$, and $g^{u}$ are all Lipschitz functions, and they have Lipschitz constants on all of $\mathscr{E}$ which are bounded by

$$
l_{g}=4 \max \left(l_{s}(r), l_{c}(r), l_{u}(r)\right) .
$$

Note that the functions $g^{s, c, u}$ defined above are not smooth. In fact, there exist Banach spaces on which there exists no smooth function $\chi(z)$, such that $\chi(z)=0$ if $\|z\|>1$, and $\chi(z)=1$ if $\|z\|<1 / 2$. If we wish to prove the existence of smooth center manifolds, we must assume that $\mathscr{E}$ has the $\mathscr{C}^{m}$ extension property, which guarantees that there exists a $\mathscr{C}^{m}$ function $\chi: \mathscr{E} \rightarrow \mathbf{R}$, which has these properties. We then redefine the functions $g^{s, c, u}=f^{s, c, u}(z) \chi(z / r)$, and set $l_{g}=2 c_{\chi} \max \left(l_{s}(r), l_{c}(r), l_{u}(r)\right)$, where $c_{\chi}$ is the Lipschitz constant of $\chi$.

Define

$$
\begin{aligned}
\mathscr{G}_{h}(\psi)_{t} & =e^{A^{c s t}}+\int_{0}^{t} d \tau e^{A^{c s}(t-\tau)} g_{h}^{c s} \circ \psi_{\tau} \\
& =\left(\begin{array}{c}
e^{A^{c_{t}}}+\int_{0}^{t} d \tau e^{A^{c(t-\tau)}} g_{h}^{c} \circ \psi_{\tau} \cdot \\
e^{A^{s_{t}}}+\int_{0}^{t} d \tau e^{A^{s(t-\tau)}} g_{h}^{s} \circ \psi_{\tau}
\end{array}\right),
\end{aligned}
$$


where $g_{h}(\xi)=g(\xi, h(\xi))$. We are going to show, under conditions which will appear as the estimates go on, that $\mathscr{G}_{h}$ has a unique fixed point in $K_{\sigma, \beta}$.

We consider $\psi \in K_{\sigma, \beta}$, and $h \in H_{\sigma}$, and we fix $\xi, \widetilde{\xi} \in \mathscr{E} c$. Applying the definitions, we find

$$
\begin{gathered}
\left\|\psi_{\tau}(\xi)-\psi_{\tau}(\tilde{\xi})\right\|_{c s} \leqq \sigma e^{\beta \tau}\|\xi-\tilde{\xi}\|_{c s} \\
\left\|h\left(\psi_{\tau}(\xi)\right)-h\left(\psi_{\tau}(\tilde{\xi})\right)\right\|_{u} \leqq \sigma^{2} e^{\beta \tau}\|\xi-\tilde{\xi}\|_{c s} .
\end{gathered}
$$

Now let

$$
w_{h, \psi_{t}}(\xi)=\psi_{t}(\xi) \oplus h\left(\psi_{t}(\xi)\right)
$$

Because of the global Lipschitz estimates on $g^{c s}$, one has

$$
\begin{aligned}
\left\|g^{c s}\left(w_{h, \psi_{t}}(\xi)\right)-g^{c s}\left(w_{h, \psi_{t}}(\tilde{\xi})\right)\right\|_{c s} & \leqq l_{g}\left\|w_{h, \psi_{t}}(\xi)-w_{h, \psi_{t}}(\tilde{\xi})\right\|_{c s} \\
& \leqq \sigma^{2} l_{g} e^{\beta t}\|\xi-\tilde{\xi}\|_{c s} .
\end{aligned}
$$

If we now project onto the center direction and integrate over $\tau$, we get

$$
\left\|P_{c} \mathscr{G}_{h}(\psi)_{t}(\xi)-P_{c} \mathscr{G}_{h}(\psi)_{t}(\tilde{\xi})\right\|_{c} \leqq\left(a\left(1+t^{k}\right)+a \sigma^{2} l_{g} \int_{0}^{t} d \tau\left(1+(t-\tau)^{k}\right) e^{\beta \tau}\right)\|\xi-\tilde{\xi}\|_{c s}
$$

and hence

$$
e^{-\beta t}\left\|P_{c} \mathscr{G}_{h}(\psi)_{t}(\xi)-P_{c} \mathscr{G}_{h}(\psi)_{t}(\tilde{\xi})\right\|_{c} \leqq\left(a \beta^{-k}(k !+1)+a \sigma^{2} l_{g} \beta^{-k-1}(k !+1)\right)\|\xi-\tilde{\xi}\|_{c s} .
$$

Similarly, we find

$$
\left\|P_{s} \mathscr{G}_{h}(\psi)_{t}(\xi)-P_{s} \mathscr{G}_{h}(\psi)_{t}(\tilde{\xi})\right\|_{s} \leqq D\left(e^{-\lambda_{s} t}+\sigma^{2} l_{g} \int_{0}^{t} d \tau e^{-\lambda_{s}(t-\tau)+\beta \tau}\right)\|\xi-\tilde{\xi}\|_{c s} .
$$

Assume now

C1:

$$
\frac{a(k !+1)}{\beta^{k}}\left(1+\frac{1}{\beta} \sigma^{2} l_{g}\right)<\sigma .
$$

This implies

$$
\left\|P_{c} \mathscr{G}_{h}(\psi)_{t}(\xi)-P_{c} \mathscr{G}_{h}(\psi)_{t}(\tilde{\xi})\right\|_{c} \leqq \sigma e^{\beta t}\|\xi-\tilde{\xi}\|_{c s} .
$$

Similarly, if we assume

C2:

$$
D+\frac{D}{\left(\lambda_{s}+\beta\right)} \sigma^{2} l_{g}<\sigma
$$

then we see that

$$
\left\|P_{s} \mathscr{G}_{h}(\psi)_{t}(\xi)-P_{s} \mathscr{G}_{h}(\psi)_{t}(\tilde{\xi})\right\|_{s} \leqq \sigma e^{\beta t}\|\xi-\tilde{\xi}\|_{c s}
$$

We have now shown that C1-C2 imply (A.9) and (A.10), which in turn imply that $\mathscr{G}_{h}$ maps $K_{\sigma, \beta}$ into itself.

We next show that $\mathscr{G}_{h}$ is a contraction. Assume $\psi$ and $\tilde{\psi}$ are in $K_{\sigma, \beta}$, and $\xi \in \mathscr{E}$ cs. By the definition of $K_{\sigma, \beta}$, we have

$$
\begin{gathered}
\left\|\psi_{\tau}(\xi)-\tilde{\psi}_{\tau}(\xi)\right\|_{c s} \leqq d_{K}(\psi, \tilde{\psi}) \cdot\|\xi\|_{c s} e^{\beta \tau} \\
\left\|h\left(\psi_{\tau}(\xi)\right)-h\left(\tilde{\psi}_{\tau}(\xi)\right)\right\|_{u} \leqq \sigma d_{K}(\psi, \tilde{\psi}) \cdot\|\xi\|_{c s} e^{\beta \tau}
\end{gathered}
$$


Substituting this into $g^{c s}$, and using the global Lipschitz estimate on $g$ we find,

$$
\begin{aligned}
\left\|g^{c s}\left(w_{h, \psi_{t}}(\xi)\right)-g^{c s}\left(w_{h, \tilde{\psi}_{t}}(\xi)\right)\right\|_{c s} & \leqq l_{g}\left\|w_{h, \psi_{t}}(\xi)-w_{h, \tilde{\psi}_{t}}(\xi)\right\|_{c s} \\
& \leqq \sigma l_{g} e^{\beta t}\|\xi\|_{c s} d_{K}(\psi, \widetilde{\psi}) .
\end{aligned}
$$

We now integrate over $\tau$ as above. Noting that the inhomogeneous term drops out, and using the general assumption $\beta>0$, we find

$$
\begin{gathered}
\left\|P_{c} \mathscr{G}_{h}(\psi)_{t}(\xi)-P_{c} \mathscr{G}_{h}(\tilde{\psi})_{t}(\xi)\right\|_{c} \leqq \frac{a(k !+1)}{\beta^{k+1}} \sigma l_{g} \cdot\|\xi\|_{c s} e^{\beta t} d_{K}(\psi, \tilde{\psi}), \\
\left\|P_{s} \mathscr{G}_{h}(\psi)_{t}(\xi)-P_{s} \mathscr{G}_{h}(\tilde{\psi})_{t}(\xi)\right\|_{s} \leqq \frac{D}{\lambda_{s}+\beta} \sigma l_{g} \cdot\|\xi\|_{c s} e^{\beta t} d_{K}(\psi, \tilde{\psi}) .
\end{gathered}
$$

Thus, we see that if

$$
\begin{gathered}
\frac{a(k !+1)}{\beta^{k+1}} \sigma l_{g}<1, \\
\frac{D}{\left(\lambda_{s}+\beta\right)} \sigma l_{g}<1,
\end{gathered}
$$

then $\mathscr{G}_{h}$ is a contraction on $K_{\sigma, \beta}$. Note that (A.11) is implied by C1 and (A.12) is implied by $\mathrm{C} 2$. Thus, we have shown the

Lemma A.2. If $h \in H_{\sigma}$, if $\sigma>1$, and if $\mathrm{C} 1-\mathrm{C} 2$ hold, then $\mathscr{G}_{h}$ has a unique fixed point $\Psi^{h}$ in $K_{\sigma, \beta}$.

Next consider the transformation

$$
\mathscr{F}(h)=-\int_{0}^{\infty} d \tau e^{-A^{u_{\tau}}} g_{h}^{u} \circ \Psi_{\tau}^{h} .
$$

We want to show that $\mathscr{F}$ maps $H_{\sigma}$ into itself and is a contraction. Let $\xi, \tilde{\xi} \in \mathscr{E}$ cs and let $h \in H_{\sigma}$. By Lemma A.2, we know that $\Psi^{h}$ is in $K_{\sigma, \beta}$. Therefore, we get, as in (A.6)

$$
\left\|g_{h}^{u} \circ \Psi_{\tau}^{h}(\xi)-g_{h}^{u} \circ \Psi_{\tau}^{h}(\tilde{\xi})\right\|_{u} \leqq \sigma^{2} l_{g} e^{\beta \tau}\|\xi-\tilde{\xi}\|_{c s} .
$$

Integrating now as in (A.7), we find

provided

$$
\|\mathscr{F}(h)(\xi)-\mathscr{F}(h)(\tilde{\xi})\|_{u} \leqq \frac{D}{\lambda_{u}-\beta} \sigma^{2} l_{g}\|\xi-\tilde{\xi}\|_{c s},
$$

C3:

$$
\lambda_{u}>\beta>0 .
$$

Thus, $\mathscr{F}$ maps $H_{\sigma}$ to itself, provided

C4:

$$
\frac{D}{\lambda_{u}-\beta} \sigma^{2} l_{g}<\sigma .
$$

We next find conditions which make $\mathscr{F}$ a contraction. Let $\xi \in \mathscr{E} c s$ and let $h, \tilde{h} \in H_{\sigma}$. We begin by bounding $P_{c}\left(\Psi_{t}^{h}(\xi)-\Psi_{t}^{\tilde{h}}(\xi)\right)$, which we rewrite as

$$
\int_{0}^{t} d \tau e^{A^{c}(t-\tau)}\left(g_{h}^{c} \circ \Psi_{\tau}^{h}(\xi)-g_{\tilde{h}}^{c} \circ \Psi_{\tau}^{\tilde{h}}(\xi)\right)
$$


(This is the usual technique of recovering the "lost derivative" after application of the contraction mapping principle.) We then write

$$
g_{h}^{c} \circ \Psi_{\tau}^{h}(\xi)-g_{\tilde{h}}^{c} \circ \Psi_{\tau}^{\tilde{h}}(\xi)=\left(g_{h}^{c} \circ \Psi_{\tau}^{h}(\xi)-g_{\tilde{h}}^{c} \circ \Psi_{\tau}^{h}(\xi)\right)+\left(g_{\tilde{h}}^{\mathcal{c}} \circ \Psi_{\tau}^{h}(\xi)-g_{\tilde{h}}^{c} \circ \Psi_{\tau}^{\tilde{h}}(\xi)\right) \text {. }
$$

Using the Lipschitz estimates

$$
\begin{gathered}
\left\|\Psi_{\tau}^{h}(\xi)\right\|_{c s} \leqq \sigma e^{\beta \tau}\|\xi\|_{c s}, \\
\left\|h\left(\Psi_{\tau}^{h}(\xi)\right)-\tilde{h}\left(\Psi_{\tau}^{h}(\xi)\right)\right\|_{u} \leqq d_{H}(h, \tilde{h}) \sigma e^{\beta \tau}\|\xi\|_{c s}, \\
\left\|\Psi_{\tau}^{h}(\xi)-\Psi_{\tau}^{\tilde{h}}(\xi)\right\|_{c s} \leqq d_{K}\left(\Psi^{h}, \Psi^{\tilde{h}}\right) e^{\beta \tau}\|\xi\|_{c s}
\end{gathered}
$$

this may be bounded by

$$
\left\|g_{h}^{c} \circ \Psi_{\tau}^{h}(\xi)-g_{\tilde{h}}^{c} \circ \Psi_{\tau}^{\tilde{h}}(\xi)\right\|_{c} \leqq\left(d_{H}(h, \tilde{h})+d_{K}\left(\Psi^{h}, \Psi^{\tilde{h}}\right)\right) l_{g} \sigma\|\xi\|_{c s} e^{\beta \tau}
$$

If we integrate, this leads to the bound

$$
\left\|P_{c} \mathscr{G}_{h}\left(\Psi^{h}\right)_{t}-P_{c} \mathscr{G}_{\tilde{h}}\left(\Psi^{\tilde{h}}\right)_{t}\right\|_{c} \leqq \frac{a(k !+1)}{\beta^{k+1}}\left(d_{H}(h, \tilde{h})+d_{K}\left(\Psi^{h}, \Psi^{\tilde{h}}\right)\right) l_{g} \sigma e^{\beta t}\|\xi\|_{c s} .
$$

The $s$-component is bounded similarly by

$$
\left\|P_{s} \mathscr{G}_{h}\left(\Psi^{h}\right)_{t}-P_{s} \mathscr{G}_{\tilde{h}}\left(\Psi^{\tilde{h}}\right)_{t}\right\|_{s} \leqq \frac{D}{\lambda_{s}+\beta}\left(d_{H}(h, \tilde{h})+d_{K}\left(\Psi^{h}, \Psi^{\tilde{h}}\right)\right) l_{g} \sigma e^{\beta t}\|\xi\|_{c s} .
$$

Combining the two bounds, we get

$$
d_{K}\left(\Psi^{h}, \Psi^{\tilde{h}}\right) \leqq\left(\frac{a(k !+1)}{\beta^{k+1}}+\frac{D}{\lambda_{s}+\beta}\right) l_{g} \sigma\left(d_{H}(h, \tilde{h})+d_{K}\left(\Psi^{h}, \Psi^{\tilde{h}}\right)\right) .
$$

Assume now

C5:

$$
\left(\frac{a(k !+1)}{\beta^{k+1}}+\frac{D}{\lambda_{s}+\beta}\right) \sigma l_{g}<1 / 2
$$

Then (A.14) implies

$$
d_{K}\left(\Psi^{h}, \Psi^{\tilde{h}}\right) \leqq L_{h} d_{H}(h, \tilde{h})
$$

where, by C5,

$$
L_{h}=2\left(\frac{a(k !+1)}{\beta^{k+1}}+\frac{D}{\lambda_{s}+\beta}\right) l_{g} \sigma<1
$$

This implies that

$$
\begin{aligned}
\left\|h\left(\Psi_{t}^{h}(\xi)\right)-\tilde{h}\left(\Psi_{t}^{\tilde{h}}(\xi)\right)\right\|_{c s} & =\left\|h\left(\Psi_{t}^{h}(\xi)\right)-h\left(\Psi_{t}^{\tilde{h}}(\xi)\right)+h\left(\Psi_{t}^{\tilde{h}}(\xi)\right)-\tilde{h}\left(\Psi_{t}^{\tilde{h}}(\xi)\right)\right\|_{c s} \\
& \leqq \sigma\left\|\Psi_{t}^{h}(\xi)-\Psi_{t}^{\tilde{h}}(\xi)\right\|_{c s}+\left\|\Psi_{t}^{\tilde{h}}(\xi)\right\|_{c s} d_{H}(h, \tilde{h}) \\
& \leqq(1+1) \sigma d_{H}(h, \tilde{h}) e^{\beta t}\|\xi\|_{c s} .
\end{aligned}
$$

Since $g^{u}$ is Lipschitz, we find that $\mathscr{F}$ is a contraction if C6:

$$
\frac{2 D}{\lambda_{u}-\beta} l_{g} \sigma<1 \text {. }
$$

Thus, we have shown 
Lemma A.3. If $\mathrm{C} 1-\mathrm{C} 6$ hold, then $\mathscr{F}$ is a contraction on $H_{\sigma}$ and has a unique fixed point in $\mathrm{H}_{\sigma}$.

Remark. From the calculations leading up to (A.2) and (A.3), we see that the graph of the fixed point, $h$, gives a center-stable manifold for (A.1).

This fixed point gives us a center-stable manifold. We now restrict the differential equation to this manifold and consider the evolution of the reduced form of (A.1) for $t \leqq 0$, or equivalently we replace $t$ by $-t$. With this change the equations take the form

$$
\begin{aligned}
& \frac{d z^{c}}{d t}=-A^{c} z^{c}-f^{c}\left(z^{c}, z^{s}, h\left(z^{c}, z^{s}\right)\right), \\
& \frac{d z^{s}}{d t}=-A^{s} z^{s}-f^{s}\left(z^{c}, z^{s}, h\left(z^{c}, z^{s}\right)\right) .
\end{aligned}
$$

To emphasize the similarity with the previous situation, define $\tilde{A}^{u}=-A^{s}$, $\tilde{f}^{u}\left(z^{c}, z^{s}\right)=-f^{s}\left(z^{c}, z^{s}, h\left(z^{c}, z^{s}\right)\right)$ and $\tilde{f}^{c}\left(z^{c}, z^{s}\right)=-f^{c}\left(z^{c}, z^{s}, h\left(z^{c}, z^{s}\right)\right)$ so that (A.16) becomes

$$
\begin{aligned}
& \frac{d z^{c}}{d t}=-A^{c} z^{c}+\tilde{f}^{c}\left(z^{c}, z^{s}\right), \\
& \frac{d z^{s}}{d t}=\tilde{A}^{u} z^{s}+\tilde{f}^{u}\left(z^{c}, z^{s}\right) .
\end{aligned}
$$

Thus, restricted to the center-stable manifold, (and with $t$ replaced by $-t$ ) the equation has a "center" part and an "unstable" part, but no "stable" part.

Note that $\tilde{A}^{u}$ defines a continuous semigroup for $t \geqq 0$, with $\sup e^{\lambda_{s} t}\left\|e^{-\tilde{A}^{u} t}\right\|=$ $\sup e^{\lambda_{s} t}\left\|e^{A^{s} t}\right\| \leqq D$, while the Lipschitz constants for $\tilde{f}^{c}$ and $\tilde{f}^{u}$, are $t \geqq 0$

$$
\tilde{l}_{c}(r)=(1+\sigma) l_{c}(r), \quad \tilde{l}_{u}(r)=(1+\sigma) l_{s}(r),
$$

by the bounds on $h$. Thus, if conditions analogous to C1-C6 with $l_{g}$ replaced by $(1+\sigma) l_{g}$ and $s$ exchanged with $u$ hold, then (A.17) will have a center-stable manifold. This manifold has no "stable" directions, and will give a center manifold for the original equation. For completeness, we state the conditions, and simplify them somewhat. Let $\lambda=\min \left(\lambda_{s}, \lambda_{u}\right)$. Then we require

C1':

$$
\frac{a(k !+1)}{\beta^{k}}\left(1+\frac{1}{\beta} \sigma^{2} l_{g}(1+\sigma)\right)<\sigma,
$$

$\mathrm{C} 2^{\prime}$ :

$$
D+\frac{D}{(\lambda+\beta)} \sigma^{2} l_{g}(1+\sigma)<\sigma,
$$

C3':

$$
\lambda>\beta>0
$$

C4':

$$
\frac{D}{\lambda-\beta} \sigma l_{g}(1+\sigma)<1 / 2 \text {, }
$$

C5':

$$
\left(\frac{a(k !+1)}{\beta^{k+1}}+\frac{D}{\lambda+\beta}\right) \sigma l_{g}(1+\sigma)<1 / 2 \text {. }
$$


These conditions are stronger than $\mathrm{C} 1-\mathrm{C} 6$ since $l_{g}$ has been replaced by $l_{g}(1+\sigma)$. (Note that $\mathrm{C}^{\prime}$ ' implies C6.) The proof of Theorem A.1 in the Lipschitz case is complete.

We now show how one proves differentiability of the center stable manifold - the smoothness of the center manifold then follows just as above, by restricting to the center stable manifold, and reversing the time. Suppose that the non-linearity, $f^{c, s, u}$, in (A.1) is $\mathscr{C}^{2}$, and that $\mathscr{E}$ has the $\mathscr{C}^{2}$ extension property. Then the cut-off nonlinearity $g^{c, s, u}$ is also $\mathscr{C}^{2}$. Define $h^{(0)}(x)=0$, and $\psi_{t}^{(0)}(x)=e^{A^{s t} t} x$. Define $h^{(n+1)}(x)=$ $\mathscr{F}\left(h^{(n)}\right)(x)$, and $\psi_{t}^{(n+1)}(x)=\mathscr{G}_{h^{(n)}}\left(\psi^{(n)}\right)_{t}(x)$. The contraction mapping theorem guarantees that $\lim _{n \rightarrow \infty} h^{(n)}(x)=h(x)$. Furthermore, from the hypothesis $\mathrm{H} 2$, there exists a constant $a_{1}$, such that

$$
\left\|e^{A^{c s t}} x\right\|_{c s} \leqq a_{1} e^{\beta|t| / 4}\|x\|_{c s} .
$$

If we now differentiate the formulas for $h^{(n+1)}$ and $\psi_{t}^{(n+1)}$, we find that provided

$$
l_{g}<\frac{\beta}{48 a_{1}},
$$

the expressions for $\left\|D_{x} h^{(n+1)}(x)\right\|$, and $\left\|D_{x}^{2} h^{(n+1)}(x)\right\|$ are uniformly bounded, independently of $n$. (The expressions for $\left\|D_{x} \psi_{t}^{(n+1)}(x)\right\|$, and $\left\|D_{x}^{2} \psi_{t}^{(n+1)}(x)\right\|$ are also uniformly bounded (in $x$ ), independently of $n$, but depending on $t$.) Once we know that $\left\|D_{x}^{2} h^{(n+1)}(x)\right\|$ is uniformly bounded, the Arzela-Ascoli theorem implies that $h(x) \in \mathscr{C}^{1}$, and this derivative is Lipschitz.

We now restrict to the center-stable manifold defined by this $h$, and consider the (time-reversed) differential equation, (A.17). The vector field in this equation is $\mathscr{C}^{1}$, with Lipschitz first derivative, and if we repeat the above argument, we find that if $\tilde{h}_{n}$ is the sequence of approximations to the center manifold of (A.17), then provided

$$
l_{g}<\beta / 48 a_{1}(1+\sigma),
$$

the first derivatives of $\tilde{h}_{n}$, and the Lipschitz constant of $D_{x} \tilde{h}_{n}$ are uniformly bounded, independent of $n$. Just as above, this implies that $\tilde{h}=\lim _{n \rightarrow \infty} \tilde{h}_{n}$ will be $\mathscr{C}^{1}$, with Lipschitz first derivative.

If $f^{c, s, u} \in \mathscr{C}^{m+1}$, and $\mathscr{E}$ has the $\mathscr{C}^{m+1}$ extension property, we can show that $h \in \mathscr{C}^{m}$, with Lipschitz $m^{\text {th }}$ derivative by this same method. One requires only that $l_{g}$ satisfy

C6':

$$
l_{g}<\text { const. } \beta / c(m),
$$

where the combinatorial factor $c(m)$ grows like $m^{k+1}$, with $k$ the exponent in $\mathrm{H} 2$. In fact, this condition can always be met if $\mathrm{C1}^{\prime}-\mathrm{C}^{\prime}$ hold, by restricting the domain of $h$ to a sufficiently small neighborhood of the origin.

Remark. Once we know that $h$ is differentiable, evaluating the derivative at the origin gives $D_{x} h(0)=0$, so we see that $h$ is tangent to $\mathscr{E}^{c s}$ at the origin.

A.1. Application to the $S-H$ Equation. We now show how this applies to the Swift-Hohenberg equation. We choose the center subspace to be the subspace spanned by the eigenvectors corresponding to the eigenvalues $\lambda_{+1, \pm}^{-}$, i.e., the two eigenvalues whose real parts are $\mathcal{O}(\varepsilon)$. For convenience we denote them as $\lambda_{ \pm}^{c}$. The stable subspace will be the span of the eigenvectors corresponding to the remaining 
eigenvalues with negative real part while the unstable subspace is the span of the remaining eigenvectors with positive real part. Note that the stable and unstable subspaces will both intersect the $n^{\text {th }}$ sector of $\mathscr{E}$ in a two dimensional subspace, with the exception of the first sector. Denote the eigenvalues and eigenvectors in the $n^{\text {th }}$ sector by $\lambda_{ \pm}^{s, n}, \phi_{ \pm}^{s, n}$, and $\lambda_{ \pm}^{u, n}, \phi_{ \pm}^{u, n}$, respectively. We will also need the adjoint eigenvectors $\psi_{ \pm}^{s, n}$, and $\psi_{ \pm}^{u, n}$, as well.

We now rewrite (1.1) in the form (A.1). We have already bounded the non-linear term $F(X)$, in Corollary 2.2. In particular, we know that $\|F(X)\|_{l} \leqq c_{F, l}\|X\|_{l}^{3}$.

We next consider the equations for $x$-the variables in the center subspace. Let $x=\left(x_{+}, x_{-}\right)$be the coordinates introduced in Sect. 2. Then we have with $z=\left(z^{c}, z^{s}, z^{u}\right)$

$$
\dot{x}=\left(\begin{array}{c}
\lambda_{+} x_{+} \\
\lambda_{-} x_{-}
\end{array}\right)+\mathbf{P}^{c} F(z),
$$

and as in Sect. 2, $\mathbf{P}^{c}=c_{+}\left|\phi_{+}\right\rangle\left\langle\psi_{+}\left|+c_{-}\right| \phi_{-}\right\rangle\left\langle\psi_{-}\right|$. We will define

and

$$
A^{c} x=\left(\begin{array}{c}
\left(\operatorname{Im} \lambda_{+}\right) x_{+} \\
\left(\operatorname{Im} \lambda_{-}\right) x_{-}
\end{array}\right)
$$

$$
f^{c}(z)=\left(\begin{array}{c}
\operatorname{Re}\left(\lambda_{+}\right) x_{+} \\
\operatorname{Re}\left(\lambda_{-}\right) x_{-}
\end{array}\right)+\mathbf{P}^{c} F(z) .
$$

Then, using the facts

(i) $\left|\operatorname{Re} \lambda_{ \pm}\right| \leqq c \varepsilon$, for some $c>0$,

(ii) $\left\|\mathbf{P}^{c}\right\| \leqq c / \varepsilon$, for some $c>0$,

(iii) Corollary 2.2,

we have

Lemma A.4. There exist positive constants $c_{1}$ and $c_{2}$ such that the Lipschitz constant $l_{c}(r)$ for $f^{c}$ is less than or equal to $\left(c_{1} \varepsilon+c_{2} r^{2} / \varepsilon\right)$.

We now turn to the stable and unstable parts of the equations. For the stable part we choose as a basis the eigenvectors $\left\{\phi_{ \pm}^{s, n}\right\}_{n \geqq 0}$, so that the operator $A^{s}$ is diagonalized and its diagonal elements are just the eigenvalues $\left\{\lambda_{ \pm}^{s, n}\right\}$. From the asymptotic estimates on the eigenvalues in Sect. 2, we see immediately that $A^{s}$ satisfies $H 1$, and we may choose the constant $\lambda_{s}$ in that hypothesis to be $c_{s} \sqrt{\varepsilon}$, for some $c_{s}>0$.

The non-linear piece $f^{s}$ will be given by $\mathbf{P}^{s} F(z)$, and we now compute the norm of $\mathbf{P}^{s}$. We note that $\mathbf{P}^{s}=\bigoplus_{n=0}^{\infty} \mathbf{P}_{n}^{s}$, where $\mathbf{P}_{n}^{s}$ is a projection onto the eigenvectors $\phi_{ \pm}^{s, n}$ in the $n^{\text {th }}$ sector. Thus, $\left\|\mathbf{P}^{s} F(z)\right\|_{l}=\sum_{n=0}^{\infty}\left\|\mathbf{P}_{n}^{s} F(z)\right\|_{l}$. But,

$$
\left\|\mathbf{P}_{n}^{s} F(z)\right\|_{l}=\left(\sum_{j=0}^{3}\left(1+n^{2}\right)^{l}\left|\left(\mathbf{P}_{n}^{s} F(z)\right)_{n, j}\right|^{2}\right)^{1 / 2} .
$$

To compute $\left(\mathbf{P}_{n}^{s} F(z)\right)_{n, j}$, we can represent $\mathbf{P}_{n}^{s}$ explicitly, as was done for $\mathbf{P}^{c}$ in Sect. 2, as

$$
\mathbf{P}_{n}^{s}=c_{n,+}\left|\phi_{+}^{s, n}\right\rangle\left\langle\psi_{+}^{s, n}\left|+c_{n,-}\right| \phi_{-}^{s, n}\right\rangle\left\langle\psi_{-}^{s, n}\right|
$$


The constants $c_{n, \pm}=1 /\left\langle\psi_{ \pm}^{s, n}, \phi_{ \pm}^{s, n}\right\rangle$ are computed by noting (see Appendix C) that $c_{n, \pm}=p_{n}^{\prime}\left(\lambda_{ \pm}^{s, n}\right)$, where $p_{n}$ is the characteristic polynomial of $M_{n}$. Once again, using the explicit expression for $p_{n}^{\prime}$, and our asymptotic estimates on the eigenvalues $\lambda_{ \pm}^{s, n}$, one finds

Lemma A.5. There exist positive constants, $c_{3}$, and $c_{4}$, such that the normalization constants $c_{n}^{ \pm}$satisfy $\left|c_{n}^{ \pm}\right| \leqq \max \left(c_{3} / \sqrt{\varepsilon}, c_{4}\right)$.

Remark. Roughly speaking, one expects $p_{n}^{\prime}\left(\lambda_{ \pm}^{s, n}\right)$ to be proportional to the distance from $\lambda_{ \pm}^{s, n}$ to the nearest eigenvalue - this is in accord with the explicit calculation that gives Lemma A.5.

If we now allow $\mathbf{P}_{n}^{s}$ to act on $F(z)$, and use the fact that $F(z)_{n, j}=0$, if $j \neq 3$, we find that $\left|\left(\mathbf{P}_{n}^{s} F(z)\right)_{n, j}\right| \leqq c_{5} \varepsilon^{-1 / 2}\left|F(z)_{n, 3}\right|$, for some constant $c_{5}$. Inserting this into (A.19), we have

Corollary A.6. There exists a positive constant $c_{6}$ such that

$$
\left\|\mathbf{P}^{s} F(X)\right\|_{l} \leqq \frac{c_{6}}{\sqrt{\varepsilon}}\|F(X)\|_{l} .
$$

This, when combined with Corollary 2.2, implies

Lemma A.7. There exists a positive constant $d_{s}$ such that the Lipschitz constant for $f^{s}$, on the ball of radius $r$, is bounded by $d_{s} r^{2} \varepsilon^{-1 / 2}$.

By exactly analogous reasoning we find

Lemma A.8. There exists a positive constant $d_{u}$ such that the Lipschitz constant for $f^{u}$, on the ball of radius $r$, is bounded by $d_{u} r^{2} \varepsilon^{-1 / 2}$.

We now verify the hypotheses of Theorem A.1. We have rewritten (1.1) so that $\mathrm{H} 1-\mathrm{H} 3$ hold, so we only need to verify the estimates $\mathrm{C1}^{\prime}-\mathrm{C} 6^{\prime}$ on the Lipschitz constants. This is easy from the above discussion. We have, uniformly in $\varepsilon>0, \varepsilon$ small, the following bounds:

$$
\lambda=\mathcal{O}\left(\varepsilon^{-1 / 2}\right), \quad D=\mathcal{O}(1), \quad a=a_{1}=1, \quad k=0 .
$$

We choose $\beta=\lambda / 2$ and $r=\varepsilon^{(3 / 4)+\gamma}$ for some $\gamma>0$. This implies $l_{g}=\mathcal{O}(\varepsilon)+$ $\mathcal{O}\left(\varepsilon^{(1 / 2)+2 \gamma}\right)$, by Lemma A.4, Lemma A.7, and Lemma A.8. If we choose $\sigma$ of order 1 , say $\sigma=2$, then there is an $\varepsilon_{0}>0$ such that for all $\varepsilon<\varepsilon_{0}$ the inequalities $\mathrm{C1}^{\prime}-\mathrm{C}^{\prime}$ hold, for $m=1$, i.e., (A.18) holds.

This means that Theorem A.1 holds and hence we have:

Theorem A.9. Let $\gamma>0$. Then for $\varepsilon$ sufficiently small, (1.1) has a center manifold, defined on a neighborhood of the origin of size $\mathcal{O}\left(\varepsilon^{(3 / 4)+\gamma}\right)$. This center manifold is $\mathscr{C}^{1}$.

\section{Appendix B. Stationary Solutions of the Swift-Hohenberg Equation}

To make this paper self-consistent, we prove again the existence of stationary solutions of (1.1) [cf. CE1]. Since such solutions correspond to stationary points of the equation in the center manifold, this will prove hypothesis $\mathrm{H} 2$ of Sect. 4.

From (1.2) and (2.1), we see that the Fourier coefficients, $\left\{W_{n}^{0}\right\}$ of the stationary 
solution must satisfy:

$$
\left(\alpha-\left(1-\omega^{2} n^{2}\right)^{2}\right) W_{n}^{0}=\sum_{p+q+r=n} W_{p}^{0} W_{q}^{0} W_{r}^{0} .
$$

If we now rescale so that $W_{n}^{0} \rightarrow \varepsilon W_{n}^{0}$, we obtain

$$
\left(\alpha-\left(1-\omega^{2} n^{2}\right)^{2}\right) W_{n}^{0}=\varepsilon^{2} \sum_{p+q+r=n} W_{p}^{0} W_{q}^{0} W_{r}^{0} .
$$

Define the Hilbert spaces $H_{\mathbf{C}}^{l}(\mathbf{Z})=\left\{\left.\mathbf{x} \in \mathbf{C}^{\mathbf{Z}}\left|\sum_{n \in \mathbf{Z}}\left(1+n^{2}\right)^{l}\right| x_{l}\right|^{2}<\infty\right\}$. (Then $H_{\mathbf{C}}^{0}(\mathbf{Z})=l^{2}(\mathbf{Z})$.)

Define the function $F(\alpha, \mathbf{x} ; \varepsilon)=\left(\alpha-\left(1-\omega^{2} n^{2}\right)^{2}\right) x_{n}-\varepsilon^{2} \sum_{p+q+r=n} x_{p} x_{q} x_{r}$. Then $F$ is a continuously differentiable (and hence analytic) function from $\mathbf{R} \times H_{\mathbf{C}}^{4}(\mathbf{Z}) \times \mathbf{R} \rightarrow$ $H_{\mathbf{C}}^{0}(\mathbf{Z})$.

Furthermore, if we define $\delta_{n}^{(0)}=\{1$ if $n= \pm 1 ; 0$ otherwise $\}$, and $\alpha^{*}=\left(1-\omega^{2}\right)^{2}$, then $F\left(\alpha^{*}, \delta^{(0)} ; 0\right)=0$.

Let $E^{4}=\left\{\right.$ subspace of $H_{\mathbf{C}}^{4}(\mathbf{Z})$ with $\left.x_{1}=x_{-1}=0 ; x_{n}=\bar{x}_{-n}\right\}$, and $E^{0}=\{$ subspace of $H_{\mathbf{C}}^{0}(\mathbf{Z})$ with $x_{n}=\bar{x}_{-n}$, and $\left.x_{1} \in \mathbf{R}\right\}$, and define $\tilde{F}: \mathbf{R} \times E^{4} \times \mathbf{R} \rightarrow E^{0}$ by $\tilde{F}(\alpha, \mathbf{x} ; \varepsilon)=$ $F\left(\alpha, \delta^{(0)}+\mathbf{x} ; \varepsilon\right)$. Then $F\left(\alpha^{*}, 0 ; 0\right)=0$ and

with

$$
\left(D_{\left(\alpha^{*}, 0\right)} \tilde{F}\right)\left(\begin{array}{c}
\delta \alpha \\
\delta \mathbf{x}
\end{array}\right)=(\delta \tilde{\mathbf{x}})
$$

$$
\delta \tilde{\mathbf{x}}_{n}= \begin{cases}\delta \alpha & \text { if } n= \pm 1 \\ \left(\alpha^{*}-\left(1-n^{2} \omega^{2}\right)^{2}\right) \delta x_{n} & \text { otherwise }\end{cases}
$$

From this we conclude that $\left(D_{\left(\alpha^{*}, 0\right)} \tilde{F}\right)$ is an invertible linear map from $\mathbf{R} \times E^{4}$ to $E^{0}$ so by the implicit function theorem, for $\varepsilon$ sufficiently small, there exists $\alpha(\varepsilon)$ and $\mathbf{x}(\varepsilon)$, with $\widetilde{F}\left(\alpha(\varepsilon), \delta^{(0)}+\mathbf{x}(\varepsilon), \varepsilon\right)=0$. Thus, $\left\{\delta^{(0)}+\mathbf{x}(\varepsilon)\right\}$ are the Fourier coefficients of a stationary solution of (1.1), and the conditions on $E^{4}$ insure that this solution is real.

Remark. This argument only implies that the stationary solution is in $\mathscr{C}^{3}$, but standard "boot-strap" arguments allow one to immediately conclude that the solution is in fact $\mathscr{C}^{\infty}$.

Remark. Note that these stationary solutions correspond to fixed points of the vector field restricted to the center manifold. Furthermore, if one unravels the various scalings and changes of coordinates, one finds that as $\varepsilon \rightarrow 0$, the stationary solutions approach the fixed point $p=0, q=\sqrt{\alpha_{0} / 3}$, of Sect. 4 .

Remark. Due to the translation invariance of the Swift-Hohenberg equation we obtain a whole family of stationary solutions by replacing $\delta^{(0)}$ in the construction above by $\delta^{\phi}=\left\{e^{i \phi}\right.$ if $n=1 ; e^{-i \phi}$ if $n=-1$; and 0 otherwise $\}$.

\section{Appendix C. Properties of the Tangent Matrix}

We summarize here a few facts about matrices of the form (2.2). We formulate them for the general $n$ by $n$ matrix which will occur for an $n^{\text {th }}$ order differential 
equation. We call the elements of the bottom row $a_{0}, \ldots, a_{n-1}$ and we define $a_{n}=-1$. Thus, the matrix is

$$
\left(\begin{array}{ccccc}
0 & 1 & 0 & \cdots & 0 \\
0 & 0 & 1 & \cdots & 0 \\
\vdots & \vdots & \vdots & \cdots & \vdots \\
0 & 0 & 0 & \cdots & 1 \\
a_{0} & a_{1} & a_{2} & \cdots & a_{n-1}
\end{array}\right) .
$$

The characteristic polynomial is

$$
p(z)=\sum_{j=0}^{n} a_{j} z^{j}
$$

If $u_{i}, i=0, \ldots, n-1$ denotes the components of an eigenvector with eigenvalue $\lambda$, then

$$
u_{i}=\lambda^{i}
$$

The components of the eigenvector of the adjoint, with eigenvalue $\bar{\lambda}$ are

$$
v_{i}=\sum_{j=i+1}^{n} \bar{a}_{j} \bar{\lambda}^{j-i-1}
$$

The inner product between these eigenvectors is

$$
\langle v \mid u\rangle=\sum_{i=0}^{n-1} \lambda^{i} \sum_{j=i+1}^{n} a_{j} \lambda^{j-i-1}=\sum_{k=1}^{n} k a_{k} \lambda^{k-1}=p^{\prime}(\lambda)
$$

Acknowledgements. The possibility of applying the center manifold methods to this problem was suggested to us by Kirchgässner and Mielke. This research was supported by the Fonds National Suisse and the NSF (grant DMS-8802118). Part of this work was done while JPE profited from the usual warm hospitality at the IHES and CEW that of the Dépt. de Physique Theorique, Univ. de Genève.

\section{References}

[A] Arnold, V.: Ordinary differential equations. Cambridge, MA: MIT Press 1973

[AW] Aronson, D., Weinberger, H.: Multidimensional nonlinear diffusion arising in Population Genetics. Adv. Math. 30, 33 (1978)

[CE1] Collet, P., Eckmann, J.-P.: The existence of dendritic fronts. Commun. Math. Phys. 107, 39-92 (1986)

[CE2] Collet, P., Eckmann, J.-P.: Instabilities and fronts in extended systems, Princeton, NJ: Princeton University Press 1990

[CM1] Carr, C., Muncaster, R. G.: The application of centre manifolds to amplitude expansions. I. Ordinary differential equations. J. Differ. Eqs. 50, 260-279 (1983)

[CM2] Carr, C., Muncaster, R. G.: The application of centre manifolds to amplitude expansions. II. Infinite dimensional Problems. J. Differ. Eqs. 50, 280-288 (1983)

[HPS] Hirsch, M., Pugh, C., Shub, M.: Invariant Manifolds: Lecture Notes in Mathematics vol. 583. Berlin Heidelberg, New York: Springer 1977 
[K] Kirchgässner, K.: Nonlinearly resonant surface waves and Homoclinic bifurcation. Adv. Appl. Mech. 26, 135-181 (1988)

[LW] de la Llave, R., Wayne, C. E.: Whiskered and Low Dimensional Tori in Nearly Integrable Hamiltonian Systems. Preprint (1989)

[M] Mielke, A.: Reduction of Quasilinear Elliptic Equations in Cylindrical Domains with Applications. Math. Meth. Appl. Sci. 10, 51-66 (1988)

Communicated by A. Jaffe 
